Nijdam MJ, Gersons BPR, Reitsma JB, de Jongh A, Olff M (2012). Brief eclectic psychotherapy v. eye movement desensitisation and reprocessing therapy in the treatment of post-traumatic stress disorder: randomised controlled trial. British Journal of Psychiatry 200, 224-231.

Paunovic N, Öst LG (2001). Cognitive-behavior therapy vs exposure therapy in the treatment of PTSD in refugees. Behaviour Research and Therapy 39, 1183-1197.

Power KG, McGoldrick T, Brown K, Buchanan R, Sharp D, Swanson V, Karatzias T (2002). A controlled comparison of eye movement desensitization and reprocessing versus exposure plus cognitive restructuring, versus waiting list in the treatment of post-traumatic stress disorder. Clinical Psychology and Psychotherapy 9, 299-318.

Powers MB, Halpern JM, Ferenschak MP, Gillihan SJ, Foa EB (2010). A meta-analytic review of prolonged exposure for posttraumatic stress disorder. Clinical Psychology Review 30, 635-641.

A. DE JONGH ${ }^{1,2}$, C. DE ROOS $^{3}$ AND I. A. E. BICANIC ${ }^{4}$

${ }^{1}$ Department of Behavioral Sciences, ACTA, University of

Amsterdam and VU University, The Netherlands

${ }^{2}$ School of Health Sciences, Salford University, Manchester, UK

${ }^{3}$ Psychotrauma Center for Children and Youth, MHO

Rivierduinen, Leiden, The Netherlands

${ }^{4}$ National Psychotrauma Center for Children and Youth,

University Medical Center Utrecht, The Netherlands

(Email: a.de.jongh@acta.nl)

Psychological Medicine, 44 (2014).

doi:10.1017/S0033291714002037

First published online 5 September 2014

\section{A rejoinder from Gerger and colleagues}

Systematic syntheses of individual trials have been described as the 'gold standard' for the evaluation of interventions (Sackett et al. 1996, p. 72). As pointed out by de Jonghe et al., systematic reviews and meta-analyses play an increasing role in the decision making of clinicians, researchers and policy makers. However, meta-analyses are, of course, not immune from bias. In their letter, de Jonghe et al. criticize our recent network meta-analysis of psychological interventions for post-traumatic stress disorder (PTSD) (Gerger et al. 2014b) for severe methodological shortcomings and question the relevance of our study. Many of the issues raised by de Jonghe et al. have already been considered in our paper. However, we would like to use the opportunity of this rejoinder to further clarify some issues.

de Jonghe et al. seem to be unsatisfied with our finding of equivalent effectiveness of specific psychological interventions. They argue that we did not identify superiority of any intervention 'due to the considerable between-trial heterogeneity', which they attribute to our inclusion of heterogeneous samples. The first part of this statement lacks scientific evidence, however. As described in the Introduction of our paper, the large majority of meta-analyses in the field of PTSD interventions conclude equivalent effectiveness of specific interventions (e.g. Bisson \& Andrew, 2007; Watts et al. 2013) and none of the interventions has consistently been shown to outperform the others; not even in meta-analyses with less between-trial heterogeneity (e.g. Benish et al. 2008). However, as stated in our Limitations we admit that we did not control for possibly moderating effects of clinical patient characteristics, which have previously been shown to affect relative effect size estimates (Gerger et al. 2014a). We have, however, conducted a moderator analysis including the status of a full PTSD diagnosis (versus subclinical PTSD symptoms) to explain heterogeneity, which de Jonghe and colleagues may have overseen in our paper.

A further point of critique is that we distinguished trials with small to moderate samples from trials with larger samples. We elaborated extensively on the rationale for the cut-offs chosen in our analyses in the Method. We are therefore not clear about the actual critique here. Our cut-offs conform to those proposed by Schnurr (2007), which also rely on power considerations. Given the vast literature on the risk of bias that is typically associated with small samples (Egger et al. 1997; Sterne et al. 2000; Cuijpers et al. 2010a, b; Nüesch et al. 2010; Barth et al. 2013; Watts et al. 2013), we do not believe that the authors aimed at fundamentally questioning the relevance of sample size as a moderator variable.

Furthermore, our conclusion of eye movement desensitization and reprocessing (EMDR) as 'promising', which is not negative in principle, seems to contradict de Jonghe et al.'s expectations. The authors argue that, in the presence of more than 20 trials on EMDR, our conclusion 'lacks scientific merit'. However, from our point of view and based on the extensive empirical literature on small sample bias, we feel very confident in repeating the conclusion regarding the lack of robust evidence for EMDR. We were unable to identify a single trial on the efficacy of EMDR that was adequately sized to detect relative intervention effects of moderate to small size. We therefore strongly argue for the need for collaborative research projects (such as the Social Phobia Psychotherapy Network by Leichsenring et al. 2009) that aim at maximizing the number of patients included in a comparative trial and at minimizing the potential for bias from researchers' preferences (the so-called allegiance bias; see Munder et al. 2011, 2012) at the same time. Our evaluation of the evidence for EMDR also mirrors the 
appraisal of the Society of Clinical Psychology (APA Division 12) that summarizes the evidence for EMDR as 'strong research support/controversial'. We admit, however, that more recent publications may reveal additional evidence from larger trials on the effectiveness of EMDR.

Referring to the last statement, de Jonghe and colleagues criticize our study for ending our search in 2010 and thereby missing 'a number of studies'. We agree that a more recent search of trials would be preferable. However, we learned from two previous updates of our meta-analysis that effect estimates and their precision changed only slightly. The main results remained constant across the updates. The reliability of the major findings regarding the equivalent effectiveness of individual types of intervention may also be regarded as confirmed by several previous metaanalyses on PTSD interventions (e.g. Bradley et al. 2005; Bisson \& Andrew, 2007) and also by the most recent meta-analysis by Watts et al. (2013). The conclusions of these meta-analyses are very similar to ours. We summarized these results in the Introduction of our paper.

We agree with de Jonghe and colleagues that our meta-analysis does not provide radically new insights with regard to the classic horse race question. However, the major advantage of our meta-analysis compared to other meta-analyses in the field of PTSD interventions is that we applied a novel approach of analysis, namely network meta-analysis. By summarizing all available evidence from studies with multiple comparisons and diverse control groups in a single analysis, network meta-analysis allowed us to address the question of relative intervention effects more elegantly and with more statistical power. Thus, our network meta-analysis allowed us to go beyond the classic horse race question and explore sources of heterogeneity and alternative ways of clustering interventions, and in particular allowed us to conduct analyses with a relatively small number of trials and a small number of direct comparisons. On closer examination of earlier meta-analyses, however, the relatively small number of seven trials, that were included in our analysis of large-scaled trials, seems to be the rule rather than the exception. Thus, in the influential systematic review by Bisson \& Andrew (2007), for instance, out of more than 80 effect size estimates only three were based on more than seven trials. The meta-analysis by Watts et al. (2013) shows a similar picture, with a large number of meta-analyses that are based on only few included trials. By combining all available evidence in one analysis we found that the so-called non-specific psychological interventions may be beneficial PTSD treatments; a finding that partly contradicts previous conclusions (e.g. Bisson \&
Andrew, 2007) but conforms to a very recent meta-analysis on present-centered therapy (Frost et al. 2014). In the latter paper, present-centered therapy, which has been designed as a control for non-specific intervention effects by Schnurr et al. (2003), has been proven to be as effective as trauma-focused and evidence-based interventions.

We therefore do not share the evaluation of de Jonghe et al. of our meta-analysis as being 'marginally' relevant 'at best'. If our findings were confirmed by high-standard trials, they may indeed be of great value for decision making in clinical practice. In this sense, as discussed in our initial report, the findings from our study, which need to be confirmed in adequately designed experimental trials, should be regarded as hypothesis generating. This, however, is true for any meta-analytic finding, as meta-analysis is observational by nature.

\section{Acknowledgments}

We thank Prof. Dr. J. Gaab for comments on a previous version of this letter.

\section{Declaration of Interest}

None.

\section{References}

Barth J, Munder T, Gerger H, Nüesch E, Trelle S, Znoj H, Jüni P, Cuijpers P (2013). Comparative efficacy of seven psychotherapeutic interventions for depressed patients: results of a network meta-analysis. PLoS Medicine 10, e1001454.

Benish SG, Imel ZE, Wampold BE (2008). The relative efficacy of bona fide psychotherapies for treating post-traumatic stress disorder: a meta-analysis of direct comparisons. Clinical Psychology Review 28, 746-758.

Bisson JI, Andrew M (2007). Psychological treatment of post-traumatic stress disorder (PTSD). Cochrane Database of Systematic Reviews. Issue 3, Art. No.: CD003388.

Bradley R, Greene J, Russ E, Dutra L, Westen D (2005). A multidimensional meta-analysis of psychotherapy for PTSD. American Journal of Psychiatry 162, 214-227.

Cuijpers P, Smit F, Bohlmeijer E, Hollon SD, Andersson G (2010a). Efficacy of cognitive-behavioural therapy and other psychological treatments for adult depression: meta-analytic study of publication bias. British Journal of Psychiatry 196, 173-178.

Cuijpers P, van Straten A, Bohlmeijer E, Hollon SD, Andersson G (2010b). The effects of psychotherapy for adult depression are overestimated: a meta-analysis of study quality and effect size. Psychological Medicine 40, 211-223.

Egger M, Smith GD, Schneider M, Minder C (1997). Bias in meta-analysis detected by a simple, graphical test. British Medical Journal 315, 629-634. 
Frost ND, Laska KM, Wampold BE (2014). The evidence for present-centered therapy as a treatment for posttraumatic stress disorder. Journal of Traumatic Stress 27, 1-8.

Gerger H, Munder T, Barth J (2014a). Specific and nonspecific psychological interventions for PTSD symptoms: a meta-analysis with problem complexity as a moderator. Journal of Clinical Psychology 70, 601-615.

Gerger H, Munder T, Gemperli A, Nüesch E, Trelle S, Jüni P, Barth J (2014b). Integrating fragmented evidence by network meta-analysis: relative effectiveness of psychological interventions for adults with post-traumatic stress disorder. Psychological Medicine. Published online: 16 April 2014. doi:10.1017/S0033291714000853.

Leichsenring F, Hoyer J, Beutel M, Herpertz S, Hiller W, Irle E, Joraschky P, Konig HH, de Liz TM, Nolting B, Pohlmann K, Salzer S, Schauenburg H, Stangier U, Strauss B, Subic-Wrana C, Vormfelde S, Weniger G, Willutzki U, Wiltink J, Leibing E (2009). The social phobia psychotherapy research network. The first multicenter randomized controlled trial of psychotherapy for social phobia: rationale, methods and patient characteristics Psychotherapy and Psychosomatics 78, 35-41.

Munder T, Flückiger C, Gerger H, Wampold BE, Barth J (2012). Is the allegiance effect an epiphenomenon of true efficacy differences between treatments? A meta-analysis. Journal of Counseling Psychology 59, 631-637.

Munder T, Gerger H, Trelle S, Barth J (2011). Testing the allegiance bias hypothesis: a meta-analysis. Psychotherapy Research 21, 670-684.

Nüesch E, Trelle S, Reichenbach S, Rutjes AWS, Tschannen B, Altman DG, Egger M, Jüni P (2010). Small study effects in meta-analyses of osteoarthritis trials: metaepidemiological study. British Medical Journal 341, c3515.

Sackett DL, Rosenberg W, Gray JA, Haynes RB, Richardson WS (1996). Evidence based medicine: what it is and what it isn't. British Medical Journal 312, 71-72.

Schnurr PP (2007). The rocks and hard places in psychotherapy outcome research. Journal of Traumatic Stress 20, 779-792.

Schnurr PP, Friedman MJ, Foy DW, Shea MT, Hsieh FY, Lavori PW, Glynn SM, Wattenberg M, Bernardy NC (2003). Randomized trial of trauma-focused group therapy for posttraumatic stress disorder: results from a Department of Veterans Affairs Cooperative Study. Archives of General Psychiatry 60, 481-489.

Sterne JAC, Gavaghan D, Egger M (2000). Publication and related bias in meta-analysis: power of statistical tests and prevalence in the literature. Journal of Clinical Epidemiology 53, 1119-1129.

Watts BV, Schnurr PP, Mayo L, Young-Xu Y, Weeks WB, Friedman MJ (2013). Meta-analysis of the efficacy of treatments for posttraumatic stress disorder. Journal of Clinical Psychiatry 74, e541-e550.

H. GERGER ${ }^{1}$, T. MUNDER ${ }^{2}$ AND J. BARTH ${ }^{3}$

${ }^{1}$ Department of Psychology, Division of Clinical Psychology and Psychotherapy, University of Basel, Switzerland

${ }^{2}$ Psychologische Hochschule Berlin, Germany

${ }^{3}$ Institute of Complementary and Integrative Medicine, University

Hospital of Zurich, Switzerland

(Email: heike.gerger@gmail.com) 